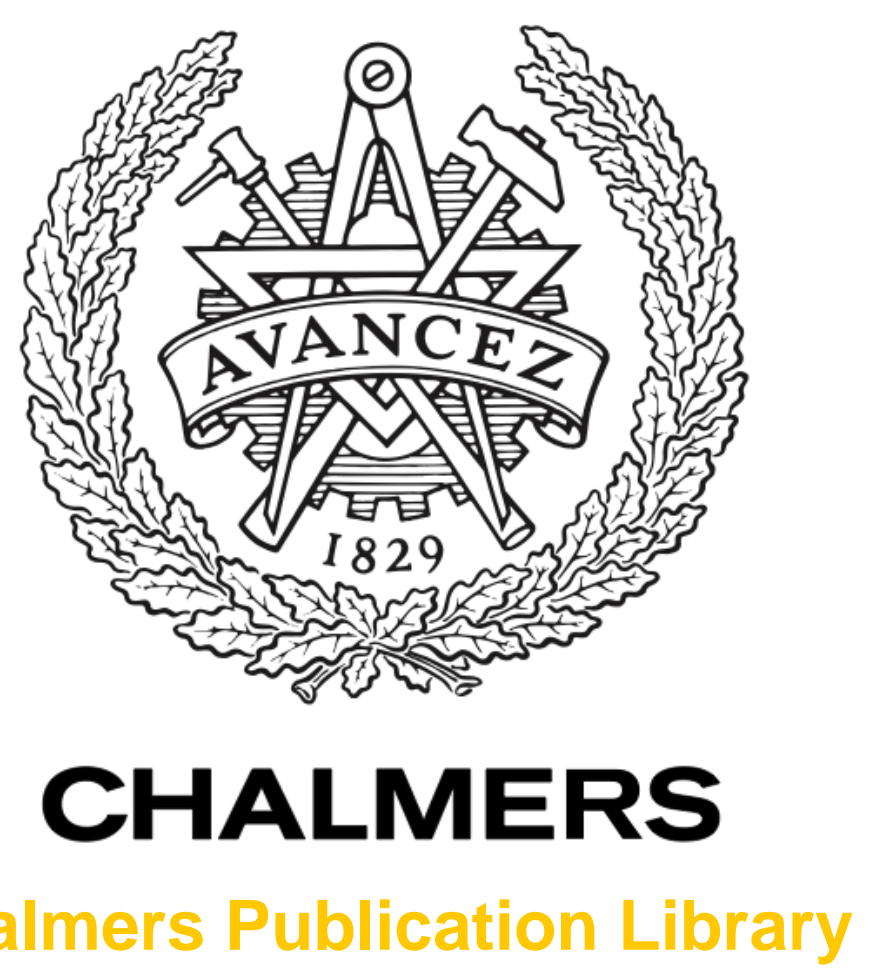

Chalmers Publication Library

\title{
Power Load and Temperature Dependence of Cold-Electron Bolometer Optical Response at $350 \mathrm{GHz}$
}

This document has been downloaded from Chalmers Publication Library (CPL). It is the author's version of a work that was accepted for publication in:

IEEE transactions on applied superconductivity (ISSN: 1051-8223)

\author{
Citation for the published paper: \\ Tarasov, M. ; Edelman, V. ; Mahashabde, S. (2014) "Power Load and Temperature \\ Dependence of Cold-Electron Bolometer Optical Response at $350 \mathrm{GHz}$ ". IEEE transactions \\ on applied superconductivity, vol. 24(6), pp. 1.
}

http://dx.doi.org/10.1109/TASC.2014.2316196

Downloaded from: http://publications.lib.chalmers.se/publication/201571

Notice: Changes introduced as a result of publishing processes such as copy-editing and formatting may not be reflected in this document. For a definitive version of this work, please refer to the published source. Please note that access to the published version might require a subscription.

Chalmers Publication Library (CPL) offers the possibility of retrieving research publications produced at Chalmers University of Technology. It covers all types of publications: articles, dissertations, licentiate theses, masters theses, conference papers, reports etc. Since 2006 it is the official tool for Chalmers official publication statistics. To ensure that Chalmers research results are disseminated as widely as possible, an Open Access Policy has been adopted.

The CPL service is administrated and maintained by Chalmers Library. 


\title{
Power Load and Temperature Dependence of Cold-Electron Bolometer Optical Response at $350 \mathrm{GHz}$
}

\author{
Mikhail A. Tarasov, Valerian S. Edelman, Sumedh Mahashabde, and Leonid S. Kuzmin
}

\begin{abstract}
Cold-electron bolometers (CEBs) integrated with twin-slot antennas have been designed and fabricated. Optical response was measured at bath temperatures of 0.06 to $3 \mathrm{~K}$ using blackbody radiation source at temperatures of 3 to $15 \mathrm{~K}$. The responsivity of $0.3 * 10^{9} \mathrm{~V} / \mathrm{W}$ was measured at $2.7-\mathrm{K}$ blackbody temperature that is close to the temperature of the cosmic microwave background. Optical measurements indicate quasioptical coupling efficiency of up to $60 \%$ at low phonon temperature and low signal level. Estimations for bolometer responsivity were made for practical range of bath temperatures and blackbody radiation temperatures. The estimated ultimate dark responsivity at $100-\mathrm{mK}$ bath temperature can approach $S_{V}=$ $10^{10} \mathrm{~V} / \mathrm{W}$ and reduces down to $1.1 * 10^{8} \mathrm{~V} / \mathrm{W}$ at $300 \mathrm{mK}$ for a device with absorber volume of $5 * 10^{-20} \mathrm{~m}^{3}$.
\end{abstract}

Index Terms-Cold-electron bolometer (CEB), electronphonon interaction, superconducting tunnel junctions, twin-slot antennas.

\section{INTRODUCTION}

C APACITIVELY coupled cold-electron bolometer (CEB) consists of a nanoabsorber and two superconductorinsulator-normal metal (SIN) tunnel junctions connected to planar antenna, schematic view, and scanning electron microscope (SEM) image shown in Fig. 1. In this bolometer, a normal metal strip, thermally isolated from the environment, is heated by incident radiation. The change in temperature is then measured by SIN junctions. The signal power is coupled to the absorber through the capacitance of tunnel junctions, dissipated in the absorber, and removed back from the absorber as hot electrons by the same SIN junctions by the bias current. Electron cooling serves as strong negative electrothermal feedback improving characteristics of the CEB, i.e., time constant,

Manuscript received December 5, 2013; revised February 14, 2014 and March 10, 2014; accepted March 12, 2014. Date of publication April 10, 2014; date of current version May 1, 2014. This work was supported in part by Swedish agencies Vetenskapsrådet, Rymdstyrelsen, the Swedish Foundation for International Cooperation in Research and Higher Education (STINT), and the Swedish Institute and the Russian Foundation for Basic Research under Grant OFIM-12145, and in part by the Ministry of Education and Science of the Russian Federation under Grant 11G34.31.0029. This paper was recommended by Associate Editor O. Mukhanov.

M. A. Tarasov is with the Vladimir Kotelnikov Institute of Radio Engineering and Electronics, 125009 Moscow, Russia (e-mail: tarasov@ @itech.cplire.ru).

V. S. Edelman is with the Peter Kapitza Institute for Physical Problems, 119334 Moscow, Russia.

S. Mahashabde and L. S. Kuzmin are with the Chalmers University of Technology, 41296 Göteborg, Sweden.

Color versions of one or more of the figures in this paper are available online at http://ieeexplore.ieee.org.

Digital Object Identifier 10.1109/TASC.2014.2316196
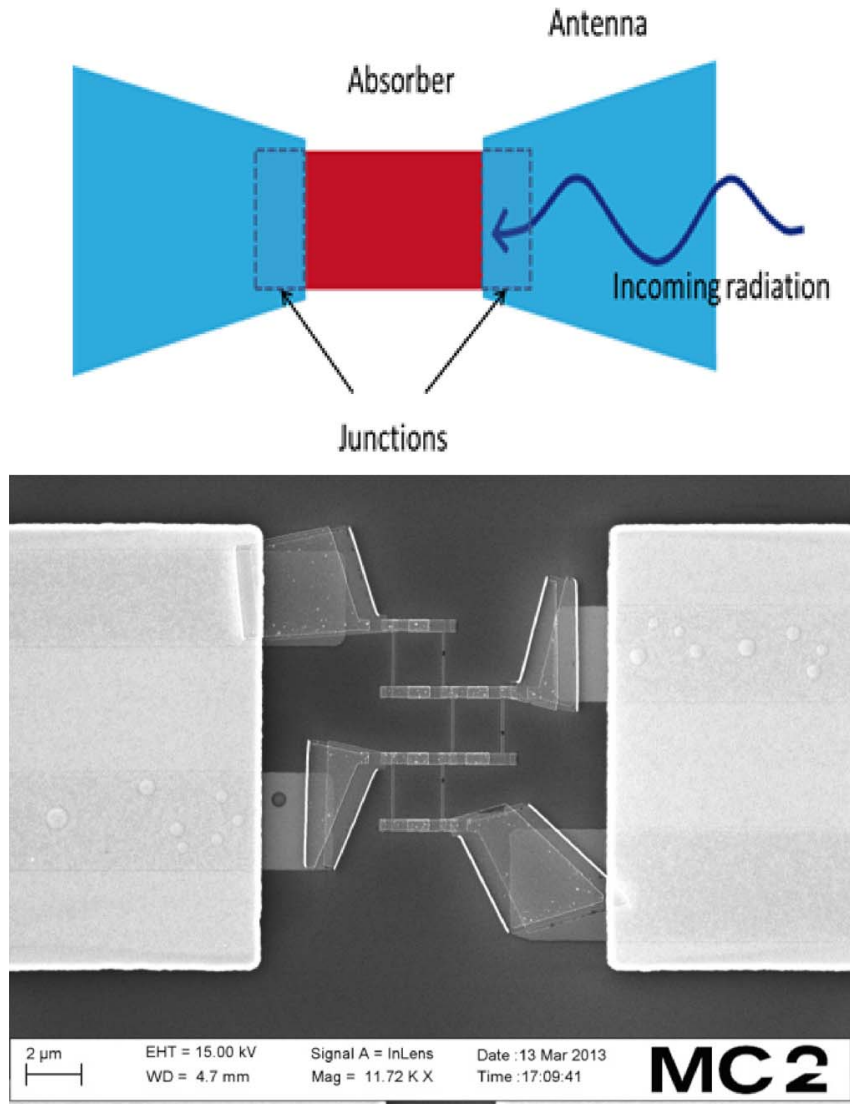

Fig. 1. Schematic view and SEM image of SINIS bolometer with a normal metal absorber, $\mathrm{Al}$ superconducting electrodes, and $\mathrm{AlO}_{x}$ tunnel barrier. Three such bolometers are connected in parallel for microwave signal and in series for output readout.

responsivity, and noise-equivalent power (NEP) [1]. Thus, CEB can be a candidate for measuring of cosmic microwave background $(\mathrm{CMB})$ radiation.

For CMB temperature of $2.7 \mathrm{~K}$, frequency of $350 \mathrm{GHz}$, and bandwidth of $100 \mathrm{GHz}$, the single-mode power load is $0.1 \mathrm{pW}$ and corresponding photon noise is $7 \mathrm{aW} / \mathrm{Hz}^{1 / 2}$. Radiation of cosmic dust clouds with temperature up to $30 \mathrm{~K}$ produces power loads up to $40 \mathrm{pW}$ corresponding to photon noise of $125 \mathrm{aW} / \mathrm{Hz}^{1 / 2}$. In our previous experiments, we studied series array of 10 CEB bolometers integrated in a cross-slot antenna [2]. In this paper, we integrated CEBs with a twin-slot antenna (see Fig. 2) and measured responsivity in $0.1-30-\mathrm{pW}$ background power range. The doubleslot antenna (DSA), also known as twin-slot antenna, is the 


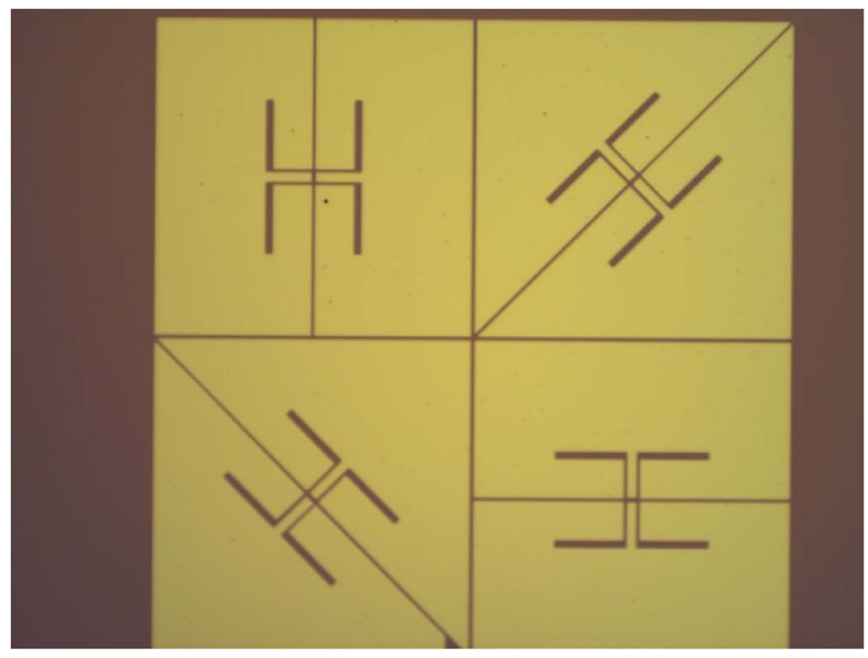

Fig. 2. Optical image of sample with four DSAs. Lines cutting antennas in halves are nonradiating at signal frequency. To avoid compromising the integrity of the ground plane, the nonradiating cuts are shunted by capacitance formed by aluminum oxide sandwiched between aluminum films, rendering these cuts invisible for submillimeter radiation.

most popular type of planar antenna that has been employed in superconductor-insulator-superconductor mixers and hotelectron bolometer mixers [3], [4]. Its spectral and beam pattern characteristics have been studied numerically and experimentally in detail, and its performance is well predicted. Apart from lens coupled slot antenna integrated bolometers, distributed antenna coupled bolometers are an alternative concept for building imaging arrays without lenses or horns for each pixel [5].

\section{EXPERIMENTAL RESULTS}

Samples with DSA (see Fig. 2) were mounted on an antireflection coated sapphire extended hyperhemispherical lens and irradiated at $350 \mathrm{GHz}$. For this experiment, a variable temperature blackbody source was fabricated in the form of a flat absorber $25 \mathrm{~mm}$ in diameter comprising $\mathrm{NiCr}$ film (300 $\Omega / \mathrm{sq}$ ) on a Si substrate. The emissivity of such film is over $90 \%$. The dc or ac current across $\mathrm{NiCr}$ film provide changing of blackbody temperature. The blackbody source was also equipped with a temperature sensor connected to the temperature controller. This allowed setting a required temperature in a range between $3 \mathrm{~K}$ and $15 \mathrm{~K}$. Due to the small size and proper choice of thermal links, such radiation source has a time constant below $100 \mathrm{~ms}$.

Radiation bandwidth was selected by two bandpass filters placed between the blackbody source and the sapphire lens. Filters were made of thick $\mathrm{Cu}$ foil; measured bandwidth is 280-380 GHz with over 20-dB suppression below $280 \mathrm{GHz}$. Each chip with bolometer structures also contained an array of 100 series-connected SIN thermometers for monitoring of phonon temperature. The sample holder was carefully shielded from stray radiation; wiring is made of twisted pairs thermally anchored on three temperature stages by metal tubes filled with mixture of Stycast epoxy and $\mathrm{Cu}$ powder that also serve as $\mathrm{RF}$ filter analog to copper powder filters usually used in dilution fridges for RF filtering. A cold container was black painted with Stycast and Bi powder mixture.

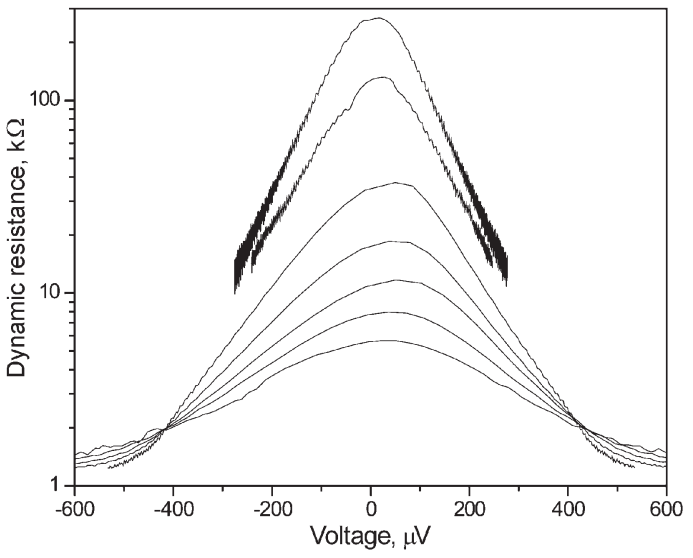

Fig. 3. Dependence values of dynamic resistance on bias voltage for phonon temperatures 274, 281, 295, 302, 309, 317, and $324 \mathrm{mK}$ (curves listed from top to bottom).

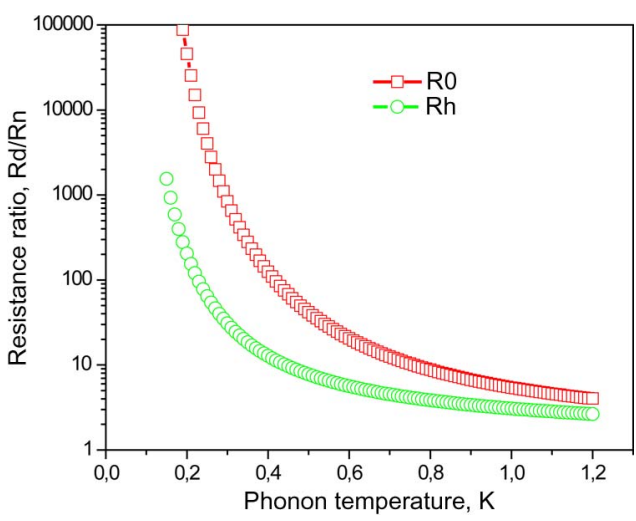

Fig. 4. RR at zero bias voltage $R_{0}$ and at half-gap voltage $R_{h}$ calculated for a pair of SIN junctions without overheating and electron cooling.

Samples with four twin-slot antennas were designed for measuring polarization of incoming radiation. The optimal size of the pixel, according to [6] is $0.5 \mathrm{~F} \lambda=0.5 * 3.5 * 0.856=$ $1.5 \mathrm{~mm}$, was designed for focal number $F=3.5$ and wavelength of $0.856 \mathrm{~mm}$. Dimension of each DSA antenna is $240 \mu \mathrm{m} \times 150 \mu \mathrm{m}$. We measured I-V curves in the bath temperature range of $0.06 \mathrm{~K}-3 \mathrm{~K}$ in dilution microcryostat [7] and from resistance ratio (RR) of SIN junction deduced equivalent electron temperature. Analytic relation for RR is given by [8]

$$
\frac{R_{d}}{R_{n}}=\sqrt{\frac{2 k T_{e}}{\pi e V_{\Delta}}} \frac{\exp \left(\frac{e V_{\Delta}}{k T_{e}}\right)}{\cosh \left(\frac{e V}{2 k T e}\right)} .
$$

Typical dependence values of dynamic resistance on bias voltage for several temperatures around $300 \mathrm{mK}$ are presented in Fig. 3, and RR calculated according to (1) is presented in Fig. 4. One can estimate the electron temperature by measuring RR for zero bias $V_{0}$, as well as measuring ratio of resistance at bias corresponding to half the superconducting gap to asymptotic resistance $R_{h}$ in Fig. 4. The latter is more reasonable because response maximum is usually observed around the half-gap voltage across the bolometer. Fig. 3 shows the dynamic resistance from measurements of one DSA sample, which is qualitatively described by relation (1) without noticeable influence of electron cooling and shunting leakage resistance. The 


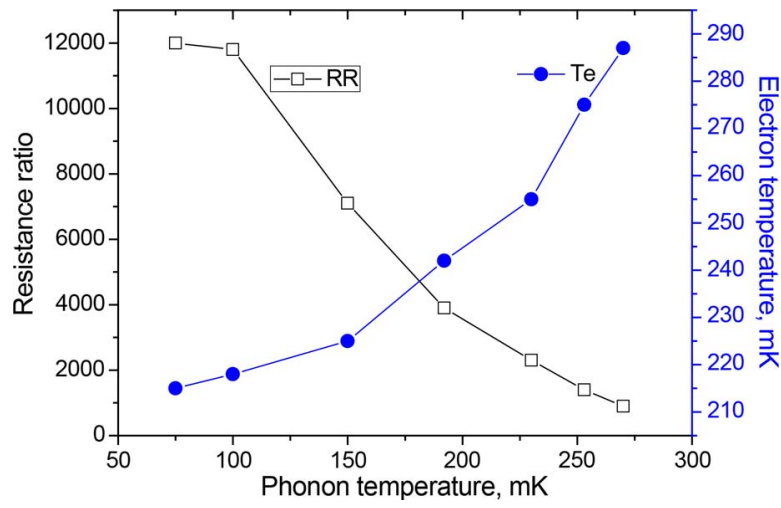

Fig. 5. RR and electron temperature $T_{e}$ dependence values on phonon temperature for dark measurements of DSA sample.

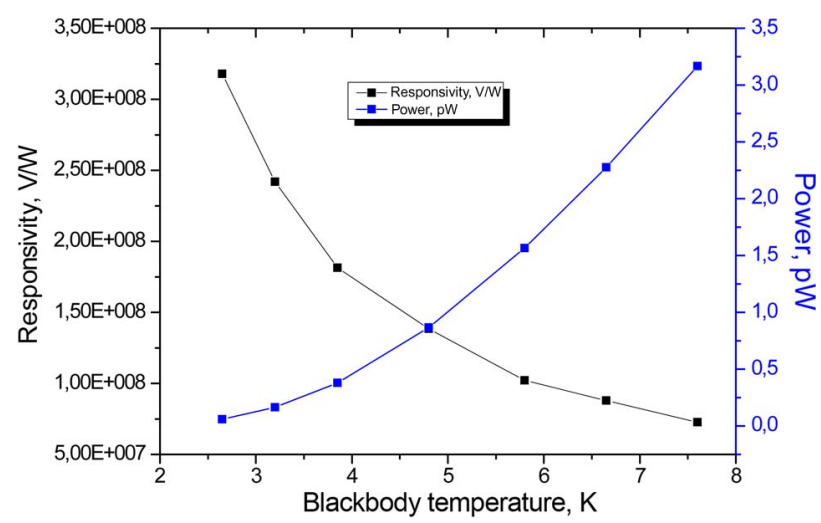

Fig. 6. Radiated power in picowatts and responsivity in microvolts per picowatt dependence values on blackbody temperature.

best obtained RR for dark measurements at $70 \mathrm{mK}$ and zero bias $(V=0)$ approaches $R_{d} / R_{n}=12000$ that corresponds to electron temperature of $215 \mathrm{mK}$ (see Fig. 5), which is a clear illustration of unavoidable overheating for our bolometers. This overheating could be explained in part by considering the recent evidence from the literature [9], which connects the Dynes density of states of the superconducting electrode to the environmental impact on the tunnel junction (normal metal-insulator-superconductor junction of the CEB in this case). We unavoidably fill the environment of the detectors with high-frequency radiation from the blackbody that could cause the decrease in the dynamic resistance of the tunnel junctions at zero bias, decreasing the RR.

For optical response measurements, we can calculate the incident power using Planck's formula where $h$ is Planck's constant, $f_{0}$ is the central frequency, $d f$ is the bandwidth, $k$ is Boltzmann's constant, $T_{R}$ is the radiation source temperature, and $\alpha$ is the emissivity.

For the single-mode DSA, antenna power irradiated at the antenna is given by

$$
P_{\text {incident }}=\frac{\alpha h f_{0} d f}{\exp \left(\frac{h f_{0}}{k T_{R}}\right)-1} .
$$

Absorbed power and corresponding electron temperature of the absorber are presented in Fig. 6. These dependence values were calculated for a signal frequency of $350 \mathrm{GHz}$, and spectral

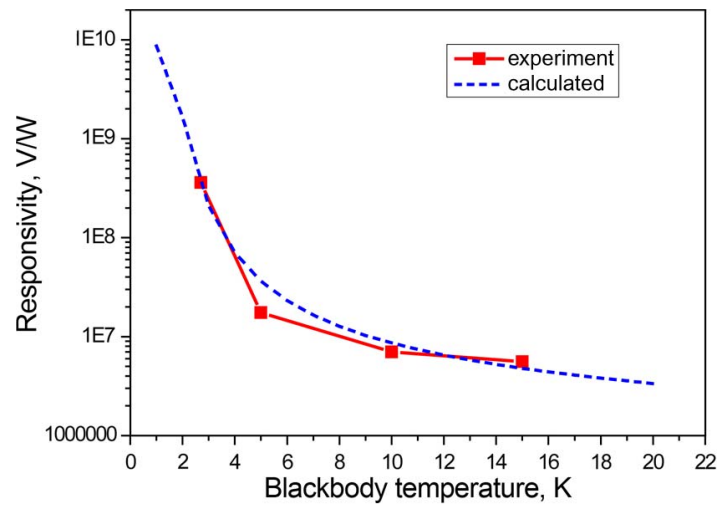

Fig. 7. Experimental and calculated voltage responsivity dependence values on blackbody radiation temperature.

transmission of bandpass, low-pass, and high-pass filters placed between radiation source and bolometer $d f=100 \mathrm{GHz}$. The illumination power value for radiation temperature of $5 \mathrm{~K}$ is $P_{\text {ins5K }}=1 \mathrm{pW}$.

Dependence values of illuminating power and responsivity on blackbody temperature are presented in Fig. 6. The absorbed power was recalculated from electron temperature of irradiated bolometer according to (3). From the measured I-V curve, we calculate dynamic resistance, compare it with what is expected for ideal SIN junction, and obtain equivalent electron temperature under irradiation. Assuming electron-phonon interaction as the main mechanism of the electron energy relaxation in bolometer

$$
P_{e p}=\Sigma \nu\left(T_{e}^{5}-T_{p}^{5}\right)
$$

where $\nu=5 * 10^{-20} \mathrm{~m}^{3}$ is the absorber volume for our sample, and $\Sigma=2 * 10^{9} \mathrm{~W} / \mathrm{m}^{-3} \mathrm{~K}^{-5}$ is the material parameter taken from [10] and [11] for the similar aluminum film with superconductivity suppressed by ferromagnetic impurities. Thus, we calculate the power absorbed in our bolometer. Ratio of incident and absorbed power corresponds to the optical efficiency of the device.

Earlier, the dependence of responsivity on dc power up to $10^{9} \mathrm{~V} / \mathrm{W}$ at $100 \mathrm{mK}$ and $10^{8} \mathrm{~V} / \mathrm{W}$ at $300 \mathrm{mK}$ was measured in [12], and here we present measurements of optical responsivity that is affected by background power load, opti$\mathrm{cal}$, and impedance mismatches. Theoretical and experimental dependence values of responsivity on radiation temperature are presented in Fig. 7. This dependence is very sharp and shows the importance of reduction for both background power level and phonon temperature. Reducing radiation temperature below $1 \mathrm{~K}$, we still observe overheating at the level of $100 \mathrm{fW}$, which comes from some background radiation and RF interferences.

We estimate optical efficiency up to 0.6 for low temperature and low signal. To obtain NEP $=30 \mathrm{aW} / \mathrm{Hz}^{1 / 2}$ for such bolometer, the phonon temperature should be below $0.2 \mathrm{~K}$, and the background radiation temperature should be below $2.5 \mathrm{~K}$. For NEP $=2 \mathrm{aW} / \mathrm{Hz}^{1 / 2}$, phonon temperature should be below $0.1 \mathrm{~K}$ and background radiation temperature should be below $1.6 \mathrm{~K}$. 


\section{DISCUSSION}

According to [13], the performance of superconductorinsulator-normal metal-insulator-superconductor (SINIS) bolometer is strongly inverse dependent on electron temperature and absorbed power. The maximum voltage responsivity in the best case at the bias point around the half of the superconducting gap voltage is

$$
S_{V}=\frac{d V}{d P}=\frac{k}{e \Sigma \nu T_{e}^{4}} .
$$

From this equation, we obtain dark responsivity $S_{d}(100 \mathrm{mK})=$ $10^{10} \mathrm{~V} / \mathrm{W}$ and $S_{d}(300 \mathrm{mK})=1.1 * 10^{8} \mathrm{~V} / \mathrm{W}$. The experimental electrical power responsivity of $10^{9} \mathrm{~V} / \mathrm{W}$ at $100 \mathrm{mK}$ obtained in [12] for dc heating of the absorber corresponds to electrical NEP $=3 \mathrm{aW} / \mathrm{Hz}^{1 / 2}$. Even lower NEP $<0.3 \mathrm{aW} / \mathrm{Hz}^{1 / 2}$ can be successfully measured with superconducting transitionedge nanobolometer at reduced levels of overheating well below $10 \mathrm{fW}$ [14], but the aim of this paper is to estimate the performance of bolometer in realistic conditions of relatively high level of background power load. We can recalculate responsivity dependence on absorbed power as

$$
S_{V}=\frac{k}{e(\Sigma \nu)^{0.2} P^{0.8}} .
$$

The calculated responsivity is $S_{V}=2 * 10^{8} \mathrm{~V} / \mathrm{W}$ at $100 \mathrm{fW}$ and $3.5 * 10^{7} \mathrm{~V} / \mathrm{W}$ at $1 \mathrm{pW}$. These estimations are made for our case of $\Sigma=2 * 10^{9} \mathrm{~W} /\left(\mathrm{m}^{3} \mathrm{~K}^{5}\right)$ and $\nu=5 * 10^{-20} \mathrm{~m}^{3}$. It means that we can expect $\mathrm{NEP}_{\mathrm{bol}}=1 \mathrm{aW} / \mathrm{Hz}^{1 / 2}$ for our amplifier noise of $10 \mathrm{nV} / \mathrm{Hz}^{1 / 2}$.

Another figure of merit for receiver performance is the level of photon noise given by

$$
\mathrm{NEP}_{\text {phot }}=(2 \mathrm{Phf})^{1 / 2} .
$$

For the presented bolometer and experimental setup, the photon noise is not observed due to limitation by amplifier noise. If the total noise is reduced down to $1 \mathrm{nV} / \mathrm{Hz}^{1 / 2}$, then photon noise could be observed at absorbed power below $0.4 \mathrm{pW}$. For single CEB with JFET readout, there is no chance to get photon noise level for power load above $1 \mathrm{pW}$ due to decrease in responsivity and a JFET voltage noise [15].

For background power load of $38 \mathrm{pW}$, the estimated responsivity is $2 * 10^{6} \mathrm{~V} / \mathrm{W}, \mathrm{NEP}_{\text {bol }}=1 \mathrm{fW} / \mathrm{Hz}^{1 / 2}$, and the corresponding photon NEP is $\mathrm{NEP}_{\text {phot }}=130 \mathrm{aW} / \mathrm{Hz}^{1 / 2}$. Hence, observation of photon noise at such power load is not achievable for single bolometer design.

The fundamental limit of thermal energy fluctuations or phonon noise is [13]

$$
\mathrm{NEP}_{\text {phonon }}=\left[10 \Sigma \nu\left(T_{e}{ }^{6}+T_{\mathrm{ph}}{ }^{6}\right)\right]^{1 / 2}
$$

where $\mathrm{NEP}_{\text {phonon }}=0.2 \mathrm{aW} / \mathrm{Hz}^{1 / 2}$ at $100 \mathrm{mK}$ and $4 \mathrm{aW} / \mathrm{Hz}^{1 / 2}$ at $280 \mathrm{mK}$, which is well below both photon and bolometer NEPs. Estimations of responsivity and NEP levels for amplifier noise of $10 \mathrm{nV} / \mathrm{Hz}^{1 / 2}$; phonon temperatures of 100,200 , and $300 \mathrm{mK}$; and power loads of $0.01-38 \mathrm{pW}$ are collected in

\begin{tabular}{|c|c|c|c|c|c|c|}
\hline $\begin{array}{l}\mathrm{T}, \mathrm{mK}, \\
\text { Power, pW }\end{array}$ & $\begin{array}{l}100 \\
0.01\end{array}$ & $\begin{array}{l}300 \\
0.01\end{array}$ & $\begin{array}{l}100 \\
0.1\end{array}$ & $\begin{array}{l}200 \\
1\end{array}$ & $\begin{array}{l}300 \\
5\end{array}$ & $\begin{array}{l}300 \\
38\end{array}$ \\
\hline Resp, V/W & $10^{10}$ & $10^{8}$ & $3 * 10^{8}$ & $3.5^{*} 10^{7}$ & $10^{7}$ & $2 * 10^{6}$ \\
\hline $\begin{array}{l}\mathrm{NEP}_{\mathrm{bol}}, \\
\mathrm{W} / \mathrm{Hz}^{1 / 2}\end{array}$ & $10^{-18}$ & $10^{-16}$ & $5 * 10^{-17}$ & $3 * 10^{-16}$ & $10^{-16}$ & $10^{-15}$ \\
\hline $\begin{array}{l}\mathrm{NEP}_{\text {photon }} \\
\mathrm{W} / \mathrm{Hz}^{1 / 2}\end{array}$ & $2 * 10^{-19}$ & $2 * 10^{-19}$ & $6^{*} 10^{-18}$ & $2 * 10^{-17}$ & $4.6^{*} 10^{-17}$ & $1.3^{*} 10^{-16}$ \\
\hline
\end{tabular}
Table I.
TABLE I

ESTIMATIONS OF RESPONSIVITY AND NEP

In practical cases, the responsivity is limited by a total power load. With the increase of absorbed power, the responsivity in (5) is reduced as $1 / P^{0.8}$. To keep it high, one can try to reduce bolometer volume, but this leads to an increase in electron temperature and finally to suppression of response even more. Increase of volume brings less suppression of response but no improvement. Responsivity can be sufficiently increased by using an array of bolometers. In this case, power absorbed in each bolometer is $N$ times less, and responsivity for each bolometer is $N^{0.8}$ times more; hence, the voltage response for single bolometer is slightly less, but total response of series array is higher. When connected in series for dc, this brings $N$ times more signal; thus, the voltage responsivity for array is

$$
S_{\text {varr }}=\frac{k N^{1.8}}{e P^{0.8}(\Sigma \nu)^{0.2}} .
$$

As example for $280-\mathrm{mK}$ phonon temperature, if we assume that electron temperature should not increase above $300 \mathrm{mK}$, it corresponds to single bolometer absorbed power of $70 \mathrm{fW}$. For 1-pW signal, this power should be distributed in 14 bolometers. For $10 \mathrm{pW}$, it should be 143 bolometers. The limiting factor for increasing the number of bolometers is the phonon power and phonon noise (7); thus, according to [15], the number of bolometers should not be more than

$$
N<\frac{P_{\mathrm{bg}}}{\Sigma \nu T_{\mathrm{ph}}^{5}} .
$$

For measurements of signal above $0.1 \mathrm{pW}$, we use arrays of bolometers connected in parallel for incoming signal and in series for readout at the output. In a previous work [2], we use ten bolometers for each polarization in a cross-slot antenna. At present, we have three bolometers in a twin-slot antenna. Topologically, it is difficult to fit more than ten bolometers in a planar antenna even at $350 \mathrm{GHz}$, and for higher frequencies, it is more challenging. Alternative to a single-mode antenna can be a focal plane array of many antennas, but this approach is facing problems of input signal matching and noise mismatch for output amplifier. Parallel connection of bolometers both for input and output with voltage bias and current readout can overcome both of these problems. In this case, a superconducting quantum interference device (SQUID) readout amplifier should be advantageous compared with a convenient JFET room-temperature amplifier. 


\section{CONCLUSION}

Voltage response of CEB integrated in a DSA has been investigated with respect to phonon temperature and power load. Estimation of dark responsivity up to $10^{10} \mathrm{~V} / \mathrm{W}$ and its decrease below $10^{7} \mathrm{~V} / \mathrm{W}$ at 5 -pW power load corresponds to experimental dependence measured with blackbody signal source at $2 \mathrm{~K}-15 \mathrm{~K}$ radiation temperatures. With amplifier voltage noise of $10 \mathrm{nV} / \mathrm{Hz}^{1 / 2}$, such responsivity corresponds to NEP from 1 to $100 \mathrm{aW} / \mathrm{Hz}^{1 / 2}$. Further improvement of CEB performance can be using focal plane array of planar antennas with single or multiple bolometers in each array element, parallel connection of such bolometers at the output, voltage bias, and SQUID readout. In such configuration, incoming power is divided between antenna array elements with bolometers, which allows preserving high responsivity by decreasing overheating for each bolometer.

\section{REFERENCES}

[1] L. Kuzmin, I. Devyatov, and D. Golubev, "Cold-electron bolometer with electronic microrefrigeration and the general noise analysis," in Proc. SPIE, 1998, vol. 3465, pp. 193-199.

[2] M. Tarasov, L. Kuzmin, V. Edelman, S. Mahashabde, and P. de Bernardis, "Optical response of a cold-electron bolometer array integrated with a 345 GHz cross-slot antenna," IEEE Trans. Appl. Supercond., vol. 21, no. 6, pp. 3635-3639, Dec. 2011.

[3] J. Zmuidzinas and H. G. LeDuc, "Quasi-optical slot antenna SIS mixers," IEEE Trans. Microw. Theory Tech., vol. 40, no. 9, pp. 1797-1804, Sep. 1992.

[4] T. M. Weller, L. P. B. Katehi, and G. M. Rebeiz, "Single and double folded-slot antennas on semi-infinite substrates," IEEE Trans. Antennas Propag., vol. 43, no. 12, pp. 1423-1428, Dec. 1995.

[5] P. Day, H. G. Leduc, C. D. Dowell, R. A. Lee, A. Turner, and J. Zmuidzinas, "Distributed antenna-coupled TES for FIR detector arrays," J. Low Temp. Phys., vol. 151, no. 1/2, pp. 477-482, Apr. 2008.

[6] M. J. Griffin, J. J. Bock, and W. K. Gear, "Relative performance of filled and feedhorn-coupled focal-plane architectures," Appl. Opt., vol. 41, no. 31, pp. 6543-6554, Nov. 2002.

[7] V. S. Edelman and G. V. Yakopov, "A dilution microcryostat cooled by a refrigerator with a pulse tube," Instrum. Exp. Tech., vol. 56, no. 5, pp. 613615, Sep. 2013.

[8] M. Nahum, P. L. Richards, and C. A. Mears, "Design analysis of a novel hot-electron microbolometer," IEEE Trans. Appl. Supercond., vol. 3, no. 1, pp. 2124-2127, Mar. 1993.

[9] J. P. Pekola, V. F. Maisi, S. Kafanov, N. Chekurov, A. Kemppinen, Yu. A. Pashkin, O.-P. Saira, M. Möttönen, and J. S. Tsai, "Environmentassisted tunneling as an origin of the Dynes density of states," Phys. Rev. Lett., vol. 105, no. 2, pp. 026803-1-026803-4, Jul. 2010.

[10] G. C. O'Neil, "Improving NIS tunnel junction refrigerators: Modeling, materials, and traps," Ph.D. dissertation, Univ. Colorado, Denver, CO, USA, 2011.

[11] J. M. Underwood, P. J. Lowell, G. C. O'Neil, and J. N. Ullom, "Insensitivity of sub-Kelvin electron-phonon coupling to substrate properties," Phys. Rev. Lett., vol. 107, no. 25, pp. 255 504-1-255 504-5, Dec. 2011.

[12] M. Nahum and J. M. Martinis, "Ultrasensitive-hot-electron microbolometer," Appl. Phys. Lett., vol. 63, no. 22, pp. 3075-3077, Nov. 1993.
[13] D. Golubev and L. Kuzmin, "Nonequilibrium theory of a hot-electron bolometer with normal-metal-insulator-superconductor tunnel junction," J. Appl. Phys., vol. 89, no. 11, pp. 6464-6472, Jun. 2001.

[14] S. Karasik, A. V. Sergeev, and D. E. Prober, "Nanobolometers for THz photon detection," IEEE Trans. Terahertz Sci. Technol., vol. 1, no. 1, pp. 97-111, Sep. 2011.

[15] L. Kuzmin, "An array of cold-electron bolometers with SIN tunnel junctions and JFET readout for cosmology instruments," J. Phys., Conf. Ser. vol. 97, no. 1, p. 012310, Feb. 2008.

Mikhail A. Tarasov received the master's degree from M. Lomonosov Moscow State University, Moscow, Russia, in 1977, and the Ph.D. and the Doctor of Sciences (Habilitation) degrees from V. Kotel'nikov Institute of Radio Engineering and Electronics of Russian Academy of Sciences, Moscow, Russia, in 1983 and 1997, respectively.

Since 1977, he has been with V.Kotel'nikov Institute, Moscow, where he is currently a Principal Investigator. For the last 20 years, he has been spending up to six months every year with Chalmers University of Technology, Gothenburg, Sweden, collaborating in development of SIS mixers, Josephson detectors, SQUID amplifiers, and cold electron bolometers. The list of his publications includes over 200 papers in scientific journals and proceedings of international conferences. His research interests are mainly in superconducting electronics, low temperature physics, microwave spectroscopy, and noise in superconducting devices.

Valerian S. Edelman was born in 1940. He received the degree from Moscow Physical and Technical Institute, Moscow, Russia, in 1963. He defended the Ph.D. degree in 1968 and the Doctor of Sciences (Habilitation) degree in 1975.

Since 1963, he has been with the staff of P. Kapitza Institute for Physical Problems of the Russian Academy of Sciences, Moscow, Russia. The main fields of scientific activity are low-temperature physics, electronic properties of metals and 2-D systems, scanning tunneling microscopy and spectroscopy, and low temperature detectors.

Since 1990, Dr. Edelman has been the Editor in Chief of the journal "Instruments and Experimental Techniques."

Sumedh Mahashabde was born in India in 1985. He received the B.E degree in instrumentation engineering from Mumbai University, Mumbai, India, in 2007, and the M.Sc. in microtechnology from Chalmers University of Technology, Gothenburg, Sweden, in 2010. He is currently working toward the Ph.D. degree in physics from Chalmers University.

His research interests include terahertz bolometers, superconducting tunnel junctions, and Graphene.

Leonid S. Kuzmin was born in Moscow, Russia, in 1946. He received the Ph.D. degree in physics (Degree of Candidate of Science in Physics and Mathematics) from Moscow State University, Moscow, Russia, in 1977, with the thesis topic "Nondegenerate single-frequency parametric amplification using Josephson junctions with self-pumping" defended in 1997 and "Correlated Tunneling of Electrons and Cooper Pairs in Ultrasmall Tunnel Junctions."

In 2000, he was a Docent with Chalmers/Göteborg University, Gothenburg, Sweden. Since 2009, he has been a Professor with Chalmers University of Technology, Gothenburg, Sweden. He is the Chairman of 13 international workshops and three international schools for young scientists with a general title "From Andreev Reflection to the Earliest Universe." He has authored more than 200 publications (including 114 in referred journals; citation index: 1305 , h-index: 19, average citations per item: 11.45). 